\title{
Processing of duplex stainless steel by WEDM
}

\author{
A. Pramanik ${ }^{1 *}$, A.K. Basak ${ }^{2}$, A.R. Dixit ${ }^{3}$, S. Chattopadhyaya ${ }^{3}$ \\ ${ }^{1}$ Department of Mech. Engineering, Curtin University, Perth, Australia \\ ${ }^{2}$ Adelaide microscopy, the University of Adelaide, Australia 5005 \\ ${ }^{3}$ Department of Mech. Eng., IIT-ISM, Dhanbad-826004, Jharkhand, India.
}

*Contacting author,

Fax: +61 (0)892662681, Phone: +61 (0)8 92667981, Email: akprama@yahoo.com; alokesh.pramanik@curtin.edu.au.

\begin{abstract}
This study investigates the manufacturing process of 2205 duplex stainless steel by wire electrical discharge machining (WEDM) where the effects of pulse-on time, wire tension and pulse-off time on surface finish, kerf width and MRR. It was found that the kerf width was unchanged with the change of pulse-on time at long pulse-of time and higher wire tension. However, it decreased initially and then increased due to the rise of pulse-on time at low values of wire tension and pulse-off time. Low wire tension and pulse-on time, pulse-off time and contributed towards widest kerf. Longer pulse-on time increased MRR due to higher machining/processing speed. Lower wire tension and shorter pulse-off time increased MRR more than that of higher wire tension and pulse-off time. Craters and recast layer were on the machined surfaces at all machining conditions. Increased pulse-on time raised surface roughness at the lower pulse-off time and tension in the wire. The surface finish at high wire tension and longer pulse-on time is always better than that at smaller pulse-on time and lower tension in the wire. The microstructure underneath the recast layer remains unchanged and the failure of wire electrode occurred at higher wire tension, longer pulse-on time and shorter pulse-off time.
\end{abstract}

Keywords: Surface; subsurface; machinability; duplex; stainless; steels; wire; edm; roughness.

\section{Nomenclatures:}


DSS $=$ Duplex stainless steel

WEDM = Wire electrical discharge machining

MRR = Material removal rate

PONT $=$ Pulse-on time

POFT $=$ Pulse-off time

$\mathrm{WT}=$ Wire tension

$\mathrm{SR}=$ Surface roughness

$\mathrm{KW}=$ Kerf width

\section{Introduction}

Among stainless steels, DSS stays in between ferrite and austenite stainless steels which combine the inherited benefits of both phases [1]. These materials have good resistance to corrosion due to the higher amount of molybdenum and/or chromium content and lower amount of sulphur content in it. The lower sulphur content also offers higher sturdiness and better high-temperature workability [2]. Higher work hardening and toughness, and lower thermal conductivity induce difficulties in machining/manufacturing stainless steels [1]. Furthermore higher resistance to fracture increases tool-chip interface temperature which generates built-up-edge at faster machining speeds and increases SR and machining forces [3, 4]. The poor machinability is also worsened by the deficiency of non-metallic additions and a lower proportion of carbon [5, 6]. More plastic austenite at higher strain causes ferrite buildup [7] which may imbalance the phase proportion during conventional machining. Therefore, non-conventional machining such as WEDM process can be applied to process these materials [8]. In wire EDM, the material is removed by consecutive electric discharges from tool electrode to the workpiece in the presence of dielectric fluid [9]. Very high temperature being generated in this method from the electrical discharge, melts the workpiece and, then the dielectric fluid at room temperature washes the debris and cools the machined surface immediately [10]. The heating cycle considerably disturbs the characteristics of these metals $[11,12]$.

The responses of materials under machining conditions comprehend the machinability of any material which also offers to understand about the important problems and emphasises main points needing fundamental attention [13-15]. Researchers applied WEDM process on different stainless steels such as, Huang et al., [16] investigated the EDM of tempered 
martensitic stainless steel at different WT, dielectric fluid pressure and power for semi-finish and finish cut. Generally, the number of machining passes depend on the required accuracy, and the required SR. Higher accuracy was achieved with several numbers of cutting pass. The machined surfaces of steel seemed to be alloyed with the material from the wire electrode. The higher diffusion of the wire materials was noted during rough machining pass. The amount of material diffusion was proportional to the cutting current [16]. Lodhi and Agarwal [17] EDMed AISI D3 steel using brass wire with zinc coating by varying current, wire feed rate, POFT and PONT and found the current contributed SR most significantly. PONT of $18 \mu \mathrm{s}$, POFT of 51 $\mu \mathrm{s}$, peak current of $180 \mathrm{~A}$ and $6 \mathrm{~mm} / \mathrm{min}$ wire feed rate were the optimum parameters for AISI D3 steel. Similarly, Sharma et al., [18] explored the influence of POFT, current, current PONT, spark gap and WT during EDM of high strength alloy steel by wire electrode made of brass. In this case the MRR was mostly influenced by spark-gap voltage, PONT and POFT. The maximum contribution on surface finish was from PONT. Higher current and PONT contribute towards lower surface finish. Lee and Tai [19] investigated the surface of D2 and H13 tool steels generated by WEDM process and found that the surface finish of both materials is the same. The surface finish increased due to the rise of PONT and current. The white layer in H13 steel is marginally thicker than that of D2 steel due to variation of thermal conductivity. The white layer thickness seems to rise due to the rise of PONT and current. Increased PONT contributes thicker white layer as well as a spike in induced stress within the material which facilitate crack formation and propagation [19]. Królczyk et al., [20] explored wear mechanisms of coated carbide tool during turning the DSS where the abrasive and adhesion wears were most prominent. They evaluated surface topography of the coated carbide tool after machining which showed that the coating of $\mathrm{Al}_{2}{ }_{2} \mathrm{O} 3$ resisted the abrasive wear significantly [21]. A mathematical model was developed to define the tool life which showed that the cutting speed was main influencing factor on the tool life [22]. The load curves and roughness profiles for various cutting wedges and variable cutting parameters were compared. The dry cutting reduced the friction for lubricated surfaces by providing a small initial contact area where the surface is contacted [23].

Based on literature survey, as briefly mentioned above, there is no investigation into the WEDM process of DSS where very high temperature is used to remove material. By taking this information in account, this study investigates the role of a number of essential input parameters towards kerf width, SR and sub-surface morphology of DSS machined by WEDM manufacturing process. This will contribute towards improve applicability of WEDM 
manufacturing process towards difficult-to-cut DSS alloys and extend the application of DSS in wider areas.

2. Materials and method

The round bar of 2205 DSS was cut into a disks of $15 \mathrm{~mm}$ thickness using a hydraulic saw. After that, both top and bottom surfaces were machined to make the sample uniform, leaving behind a clean and workable $14 \mathrm{~mm}$ thick disks. The WEDM process of 2205 DSS was performed in FANUC ROBOCUT $\propto-0 \mathrm{iD}$ instrument. All the details of experiments and variables are presented in table 1.

Table 1: Experimental variables used during WEDM process.

Average roughness of newly generated surfaces was estimated by Talysurf connected to a Mitutoyo SJ-201 remote. The machined surface was prepared by lapping or polishing and followed by chemical etching with cupric chloride solution based etchant to study the microstructures. The cylindrical cut outs from WEDM process were polished on the cylindrical surface to see the microstructural change along the depth (Fig. 1). Olympus SC100 optical microscope was used to measure the $\mathrm{KW}$ as well as to analyse microstructures. MRR was calculated from average KW, average hole diameter and an average thickness of disks and machining time.

Fig. 1: Cylindrical cut outs during machining (a) fresh part and (b) machined part with the polished and etched strip.

The input parameters during EDM were classified in 'low' and 'high' values. The 'low' values designated the lower magnitude of parameters (4 $\mu$ s PONT, $22 \mu$ s POFT and $1000 \mathrm{~kg}$ WT) except the one under assessment. Similarly, 'high' designated the higher magnitude of parameters ( $8 \mu$ s PONT, $66 \mu$ sOFT then $1800 \mathrm{~kg}$ WT) except the one under assessment. Interactions among various input parameters substantially affect the output parameters [24-26]. Hence, this effect is included in this investigation by considering high and low values of input parameters.

3. Results and discussion 
$\mathrm{KW}$ is the width of cut in the WEDM process which is identical to the diameter of wire electrode plus the double of the spark gap. Fig. 2 shows the influence of PONT on the average distance across the kerf at (a) low POFT (22 $\mu \mathrm{s})$ and WT (1000 kg), and (b) high POFT (66 $\mu \mathrm{s})$ and WT $(1800 \mathrm{~kg})$. It is interesting to note that, the influence of PONT on the KW is completely opposite when the values of the constant parameters are maximum or minimum. At low WT and POFT, the KW is high initially, subsequently a sharp decrease due to the rise of PONT and then increases again with the subsequent rise of PONT. On the other hand, the KW gradually rises with the rise of PONT and then reduces with the additional rise of PONT when the WT and POFT are high. The KW at high values of WT and POFT is always smaller than that at low values of these parameters except when the PONT is of medium value. Fig. 3 shows the influence of POFT on the KW for (a) low PONT (4 $\mu \mathrm{s})$ and tension (1000 kg), and (b) high PONT ( $8 \mu \mathrm{s})$ and tension $(1800 \mathrm{~kg})$. Initially, the KW increases gradually with the upswing of POFT then it lessens sharply with the further increase of POFT at low WT and PONT. However, the KW gradually decreases with the increase of POFT for the range considered in this study when the PONT and WT are high. It is noted that, the KW at high values of PONT and WT is smaller than that at low values of PONT and WT only when the POFT is of medium value. Fig. 4 presents the effect of WT on the KW for (a) low PONT ( $4 \mu \mathrm{s})$ and POFT (22 $\mu \mathrm{s})$, and (b) high PONT ( $8 \mu \mathrm{s})$ and POFT (66 $\mu \mathrm{s})$. A steady diminution of KW was noted with the upsurge of WT for the range considered in this study in both cases. It is noted that the KW at high values of pulse-on and POFTs is always smaller than that at low values of pulse-on and POFTs except when the WT is of low value.

Fig. 2: Effect of PONT on the KW at high and low values of constant parameters.

Fig. 3: Effect of POFT on the KW at high and low values of constant parameters.

The KW changes with machining conditions, workpiece materials and the state of wire electrode. It is well established that longer PONT generates higher heat that increases MRR and rises the KW. However, this high heat also removes the wire material and easily deforms the circular cross-section of wire to oval shape which in turn reduces the KW [8]. The final KW accounts these two facts and it reduces with the rise of PONT initially followed by a decrease with the more upsurge of PONT when the constant parameters are high [27]. There is an optimum PONT for which the kerf width is minimum in this case. Higher POFT contributes 
to reducing heat which reduces the KW. The wire remains flexible when the WT is low which contributes to larger $\mathrm{KW}$. In contrast, the rigid wire at higher tension gives reduced $\mathrm{KW}$. Therefore, the widest kerf is generated at low PONT, POFT and WT as shown in Fig. 2. The kerf generated in different machining conditions are very similar in nature.

However, those are not very straight although the electrode travelled straight vertically. The kerfs have wave-like surface formation in all the cases. Fig. 5 shows a kerf that was produced at $6 \mu \mathrm{s}$ PONT, $66 \mu \mathrm{s}$ POFT and $1800 \mathrm{~kg}$ tension in electrode. The edges of the kerf experience flaking and causes brittle failure. The uneven fractures and breakages in kerf edge many be due to high temperature and speed of the wire electrode during machining. The $\mathrm{KW}$ at bottom and top sides are not equal and result in a 'tapered cut' [8].

Fig. 4: Influence WT on the KW when constant parameters are of high and low values.

Fig. 5: Kerf generated during WEDM process of DSS at $6 \mu$ SONT, $66 \mu$ s POFT and 1800 $\mathrm{kg} \mathrm{WT}$ at (a) top and (b) bottom sides of workpiece.

Figure 6 depicts the effect of PONT on the MRR at low and high values of constant parameters. The overall MRR rises slightly with the rise of PONT in both cases for the range considered in this study. It is shown that, the MRR is much greater at low values of constant parameters compare to that at high values of constant parameters. The influence of POFT on MRR at high and low values of constant parameters is shown in Fig. 7. The figure indicates that the MRR decreases significantly initially with the upsurge of POFT then it lessens gradually with the additional intensification of POFT in both cases. The MRR is slightly higher at high values of constant parameters compare to that at low values of constant parameters. No significant variation in MRR is noted with the rise of WT at low or high values of constant parameters as depicted in the Fig. 8. It also presents that the MRR for all the WTs is much higher at low values of constant parameters compare to that at high values.

Fig. 6: Effect of PONT on the MRR at low and high values of constant parameters.

Fig. 7: Effect of POFT on the MRR at low and high values of constant parameters. 
Fig. 8: Influence of WT on MRR at low and high values of constant parameters.

Greater MRR at upper PONT is due to generation of higher temperature which increases the machining speed. The shorter POFT allows more frequent pulse-on therefore higher temperature becomes more consistence. On the other hand, though the flexible wire at lower tension produces wider kerf, it takes longer time to remove materials. Therefore, the MRR seems don't change notably with the rise of tension in electrode. Low values of WT and POFT give higher MRR compare to that of larger WT and POFT while the PONT is varied. For the same reasons, the trend of MRR can be explained by the variations of POFT and WT.

The surface of 2205 DSS generated from WEDM process at diverse machining settings is very similar. Fig. 9 presents a surface machined at $8 \mu$ sONT, $22 \mu$ s POFT and $1800 \mathrm{~kg}$ WT. It contains numerous minuscular craters related to the indiscriminate spark release from wire electrode to work-piece. Consequently, the finish of the machined surface mainly is determined by the dimensions of the spark crater. The removal of workpiece material takes place because of electric spark at many points simultaneously. The splattering of the workpiece materials occurs in the gap between workpiece and electrode where the both of these were partially coated by melted workpiece material (Fig. 9) [8]. A portion of the melted workpiece is removed by the flow of electrolyte as debris. The leftover melted metal resolidifies by quenching very fast by the flow of electrolyte and hence forward re-cast layer is generated. Therefore the appearance and finish of machined surfaces are not considerably influenced by the variation of machining conditions.

Fig. 9: Surface of 2205 DSS generated from wire EDM.

Effects of PONT, POFT and WT on the roughness of machined surfaces are presented in figures 10, 11 and 12 respectively. Fig. 10 displays that the overall roughness rises with the rise of PONT in both cases of low and high values of constant parameters. The increase of SR is significant when the constant variables are of low values i.e., low POFT $(4 \mu \mathrm{s})$ and WT (1000 $\mathrm{kg}$ ). However, the roughness rises slightly with the rise of PONT when the constant parameters have high values. It is noted that SR is higher for the lower values of the constant parameter for all PONTs except the medium value when the SR is very similar for both cases. Fig. 11 shows that, SR initially decreases slightly by the rise of POFT and then increases slightly by the further rise of POFT for both low and high values of constant parameters. In this case, the 
SR at high values of constant parameters is higher than that at low values of constant parameters for the range of POFT considered in this investigation. The effects of WT on the roughness as shown in Fig. 12 are very similar to that of PONT on the roughness which was presented in Fig. 10. In this case, the SR at low and medium values of WT is very similar at high and low values of constant parameters. However, it is reasonably higher at low values of pulse-off and PONTs than those of high values when the WT is high.

Fig. 10: Influence of PONT on SR at high and low values of constant parameters.

Fig. 11: Influence of POFT on SR at high and low values of constant parameters.

Fig. 12: Influence of WT on SR at different values of constant parameters.

When all parameters are in favour such as, shorter POFT and low WT, the rise of PONT makes bigger crater and therefore the higher SR as shown in Fig. 10. At higher WT, the wire is more rigid, and length of active cutting is shorter which may contribute to make bigger crater. This may increase roughness by the rise of WT. Therefore, the roughness at longer PONT and high WT is always higher than that at shorter PONT and lower WT for the rage of pulse on time considered in this investigation. For the similar reason, due to the adjustment of effects of POFT, PONT and WT, the variation of roughness in Fig. 12 is nonsignificant.

The microstructure of 2205 DSS before machining is presented in Fig. 13 where the light coloured ferrite phase is uniformly distributed, like islands, in the dark colour austenite phase. The proportion of these phases are almost equal. The microstructures of machined sub-surface (the location of which is shown schematically in Fig. 1 at various machining circumstances are presented in Fig. 14. The top dark part in the pictures are machined surface or recast layer. Towards the bottom of the pictures, the microstructure is exposed gradually with the increasing depth from the machined surface. The figure does not show any change in microstructure on the exposed surfaces at different conditions. The microstructures for all the presented conditions are very uniform where the proportion of austenite and ferrite seems equal at different depths from the machined surface. It is very similar to the original structure before machining (Fig. 11). 
The metallurgy of DSS is as follows, briefly: if heated to $1350{ }^{\circ} \mathrm{C}$ and hold at this point, maximum of the austenite transforms to ferrite phase, and ferrite grains form. At cooling from 1350 to $800{ }^{\circ} \mathrm{C}$, the austenite phase precipitates all over the borders of ferrite grains. The ultimate structure is determined by the factors of thermal cycles, for example, topmost temperature, time of holding, and the rate of cooling [28]. Typically, very fast rate of cooling gives remarkably coarse grains of ferrite [29]. This is disadvantageous to mechanical and corrosion behaviours $[30,31]$. Nevertheless, the transformation of appropriate quantity of austenite and exclusion of intermetallic compounds is essential to conserve the quality. In general, DSS alloys with more than around $75 \%$ ferrite content is not suitable in maximum applications. DSS has the affinity to develop intermetallic compounds of chromium, molybdenum and iron if heated to 700 to $955^{\circ} \mathrm{C}$ temperature for an extended amount of time. The intermetallic compounds reduce the toughness and corrosion resistance. These compounds are formed at a very high rate in 815 to $870{ }^{\circ} \mathrm{C}$ in DSS [32, 33].

In WEDM process, the sparks are generated in discrete points and the temperature generation is highly localised. In addition, the continuous flow of the electrolyte takes away heat and machined debris. All these do not allow to hold the machining temperature for longer period. Therefore, for the range of parameters considered in the study are not capable of changing the microstructures below the recast layer.

Fig. 13: Structure of DSS before machining.

Fig. 14: Microstructure of machined DSS at diverse conditions: (a) PONT $4 \mu \mathrm{s}$, POFT $44 \mu \mathrm{s}$, WT $1000 \mathrm{~kg}$, (b) PONT $8 \mu \mathrm{s}$, POFT $22 \mu \mathrm{s}$, WT $1400 \mathrm{~kg}$ and (c) PONT $8 \mu \mathrm{s}$, POFT $66 \mu \mathrm{s}$ and WT $1400 \mathrm{~kg}$.

Wire breakage occurred twice only at PONT $8 \mu$ s, POFT $22 \mu \mathrm{s}$ and WT $1800 \mathrm{~kg}$ during this study. In this case, the machining feed was the fastest machining run among all the experiments. There is no doubt that the generated temperature was high enough to soften the wire material that failed at relatively higher WT. The tip of the failed wire is presented in Fig. 15. It shows that necking was less in Fig. 15a compare to Fig. 15b. This indicates that the rupture of the 
wire might be abrupt as well as gradual. The unexpected failure occurs at a higher temperature, and the gradual failure occurs at lower localized temperature generated from discrete sparks.

Fig. 15: Broken tips of the wire electrode during WEDM process of 2205 DSS.

\section{Conclusions}

The manufacturing process of 2205 DSS by WEDM has been investigated in this analysis. The contribution of a number of process parameters towards surface morphology of the machined components has been investigated, which can be summarised as follows:

(a) A nonlinear trend of KW is noticed with the rise of PONT. The kerfs surface are in wavy form and contain the evidence of brittle fracture at the edges.

(b) Shorter POFT and lower WT give greater MRR than that at longer POFT and higher WT with the variation of PONT. The WT does not influence the MRR significantly. The wire electrode may rupture either gradually or suddenly due to the change of wire diameter depending on the discrete electric sparks at extreme machining conditions, where the temperature generation and WT are higher.

(c) The machined surface consists of craters and solidified molten material. The microstructure of the machined DSS below the recast layer remains unchanged which is due to very short or no holding time at high temperature during electric sparks which are discrete in nature. The increase of PONT increases the SR at shorter POFT and low WT.

(d) The wire failure occurs at longer PONT and higher WT. The longer PONT softens the wire material locally and the WT applies the stress to fail the wire.

\section{References}

[1] Nomani J, Pramanik A, Hilditch T, Littlefair G. Chip formation mechanism and machinability of wrought duplex stainless steel alloys. The International Journal of Advanced Manufacturing Technology 2015, 80(5-8), 1127-35.

[2] Renaudot N, Chauveau E, Mantel M. Machinability of duplex stainless steels long products: how to deal with the sulphur way? Revue de Métallurgie - International Journal of Metallurgy 2011, 108(4), 245-57.

[3] Nomani J, Pramanik A, Hilditch T, Littlefair G. Stagnation Zone during the Turning of Duplex SAF 2205 Stainless Steels Alloy. Materials and Manufacturing Processes. 2017, 32(13), 1486-1489,.

[4] Thiyagu M, Karunamoorthy L, Arun Kumar N. Magnetorheological fluid-based nanotexturing of tool inserts for turning of duplex stainless steel. Materials and Manufacturing Processes. 2017, 32(9), 1019-25. 
[5] Nilsson J-O. Super duplex stainless steels. Materials science and technology. 1992, 8(8), 685-700.

[6] Voronenko B. Austenitic-ferritic stainless steels: A state-of-the-art review. Metal Science and Heat Treatment. 1997, 39(10), 428-37.

[7] Nomani J, Pramanik A, Hilditch T, Littlefair G. Investigation on the behavior of austenite and ferrite phases at stagnation region in the turning of duplex stainless steel alloys. Metallurgical and Materials Transactions A. 2016, 47(6), 3165-77.

[8] Pramanik A, Littlefair G. Wire EDM Mechanism of MMCs with the Variation of Reinforced Particle Size. Materials and Manufacturing Processes. 2016, 31(13), 17001708.

[9] Pramanik, A. Electrical discharge machining of MMCs reinforced with very small particles. Materials and Manufacturing Processes. 2016, 31(4), 397-404.

[10] Mandal A, Dixit AR, Chattopadhyaya S, Paramanik A, Hloch S, Królczyk G. Improvement of surface integrity of Nimonic C 263 super alloy produced by WEDM through various post-processing techniques. The International Journal of Advanced Manufacturing Technology. 2017, 93,433-443.

[11] Pramanik A, Littlefair G, Basak A. Weldability of duplex stainless steel. Materials and Manufacturing Processes. 2015, 30(9), 1053-68.

[12] Davidson K, Singamneni S. Selective laser melting of duplex stainless steel powders: An investigation. Materials and Manufacturing Processes. 2016, 31(12), 1543-55.

[13] Hakami F, Pramanik A, Basak AK. Tool wear and surface quality of metal matrix composites due to machining: A review. Proceedings of the Institution of Mechanical Engineers, Part B: Journal of Engineering Manufacture. 2017, 231(5), 739-52.

[14] Pramanik A, Basak A, Islam MN. Effect of reinforced particle size on wire EDM of MMCs. International Journal of Machining and Machinability of Materials. 2015, 17(2), $139-49$.

[15] Vidyarthy RS, Dwivedi DK, Muthukumaran V. Optimization of A-TIG process parameters using response surface methodology. Materials and Manufacturing Processes. 2017, 1-9.

[16] Huang CA, Shih CL, Li KC, Chang Y-Z. The surface alloying behavior of martensitic stainless steel cut with wire electrical discharge machine. Applied surface science. 2006, 252(8), 2915-26.

[17] Lodhi BK, Agarwal S. Optimization of machining parameters in WEDM of AISI D3 Steel using Taguchi Technique. Procedia CIRP. 2014, 14, 194-9.

[18] Sharma N, Khanna R, Gupta R. Multi quality characteristics of WEDM process parameters with RSM. Procedia Engineering. 2013, 64, 710-9.

[19] Lee H-T, Tai TY. Relationship between EDM parameters and surface crack formation. Journal of Materials Processing Technology. 2003, 142(3), 676-83.

[20] Krolczyk G, Legutko S, Raos P. Cutting wedge wear examination during turning of duplex stainless steel. Tehnički Vjesnik - Technical Gazette. 2013, 20(3), 413-418.

[21] Krolczyk G, Gajek M, Legutko S. Effect of the cutting parameters impact onto tool life in duplex stainless steel turning process. Tehnički Vjesnik - Technical Gazette. 2013, 20(4), 587-592.

[22] Krolczyk GM, Legutko S. Experimental analysis by measurement of surface roughness variations in turning process of duplex stainless steel. Metrology and Measurement Systems. 2014, 21(4), 759-770.

[23] Krolczyk G, Gajek M, Legutko S. Predicting the tool life in the dry machining of duplex stainless steel. Eksploatacja i Niezawodnosc - Maintenance and Reliability. 2013, 15 (1), $62-65$ 
[24] Islam M, Boswell B, Pramanik A, editors. An Investigation of Dimensional Accuracy of Parts Produced by Three-Dimensional Printing. Proceedings of the World Congress on Engineering; 2013.

[24] Islam M, Anggono J, Pramanik A, Boswell B. Effect of cooling methods on dimensional accuracy and surface finish of a turned titanium part. The International Journal of Advanced Manufacturing Technology. 2013, 69(9-12), 2711-22.

[26] Islam M, Pramanik A. Effects of Insert Geometry and Feed Rate on Quality Characteristics of Turned Parts. Journal of Advanced Manufacturing Systems. 2015, 14(03), 149-66.

[27] Pramanik A, Islam MN, Boswell B, Basak AK, Dong Y, Littlefair G. Accuracy and finish during wire electric discharge machining of metal matrix composites for different reinforcement size and machining conditions. Proceedings of the Institution of Mechanical Engineers, Part B: Journal of Engineering Manufacture. 2016, DOI: 10.1177/0954405416662079.

[28] Lechartier A, Martin G, Comby S, Roussel-Dherbey F, Deschamps A, Mantel M, et al. Influence of the Martensitic Transformation on the Microscale Plastic Strain Heterogeneities in a Duplex Stainless Steel. Metallurgical and Materials Transactions A. 2017, 48(1), 20-5.

[29] Mishra M, Gunasekaran G, Rao A, Kashyap B, Prabhu N. Friction Stir Processing of 2507 Super Duplex Stainless Steel: Microstructure and Corrosion Behaviour. Friction Stir Welding and Processing IX: Springer.2017, 289-96.

[30] Wang J, Lu M-x, Zhang L, Chang W, Xu L-n, Hu L-h. Effect of welding process on the microstructure and properties of dissimilar weld joints between low alloy steel and duplex stainless steel. International Journal of Minerals, Metallurgy, and Materials. 2012, 19(6), 518-24.

[31] Zhao Y, Liu X, Li X, Wang Y, Zhang W, Liu Z. Pitting corrosion behavior in novel Mn$\mathrm{N}$ alloyed lean duplex stainless steel containing Cu. Journal of Materials Science. 2018, 53(1), 824-36.

[32] Svensson L, Gretoft B, editors. Propertiesmicrostructure relationship for duplex stainless steel weld metals. Proceedings In: Conference Duplex Stainless Steels. 1986.

[33] Li X, Li T, Lo K, Kwok C, Chen F. Influences of thermomechanical treatments on the cryogenic treatability of a slightly unstable austenitic stainless steel. Materials and Manufacturing Processes. 2017, 1-9.

Tables and figures:

Table 1: Experimental variables used during WEDM process. 


\begin{tabular}{|c|c|c|c|}
\hline Experiment no & PONT $(\mu \mathrm{s})$ & POFT $(\mu \mathrm{s})$ & WT (kg) \\
\hline 1 & 4 & 22 & 1000 \\
\hline 2 & 6 & 22 & 1000 \\
\hline 3 & 8 & 22 & 1000 \\
\hline 4 & 4 & 66 & 1800 \\
\hline 5 & 6 & 66 & 1800 \\
\hline 6 & 8 & 66 & 1800 \\
\hline 1 & 4 & 22 & 1000 \\
\hline 7 & 4 & 44 & 1000 \\
\hline 8 & 4 & 66 & 1000 \\
\hline 9 & 8 & 22 & 1800 \\
\hline 10 & 8 & 44 & 1800 \\
\hline 6 & 8 & 66 & 1800 \\
\hline 1 & 4 & 22 & 1000 \\
\hline 11 & 4 & 22 & 1400 \\
\hline 12 & 4 & 22 & 1800 \\
\hline 13 & 8 & 66 & 1000 \\
\hline
\end{tabular}




\begin{tabular}{|l|l|l|l|}
\hline 14 & 8 & 66 & 1400 \\
\hline 6 & 8 & 66 & 1800 \\
\hline
\end{tabular}

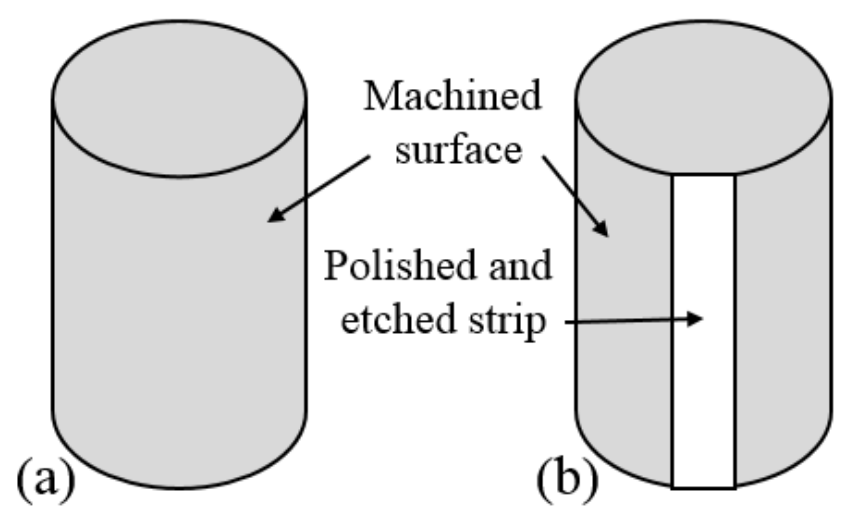

Fig. 1: Cylindrical cut outs during machining (a) fresh part and (b) machined part with the polished and etched strip.

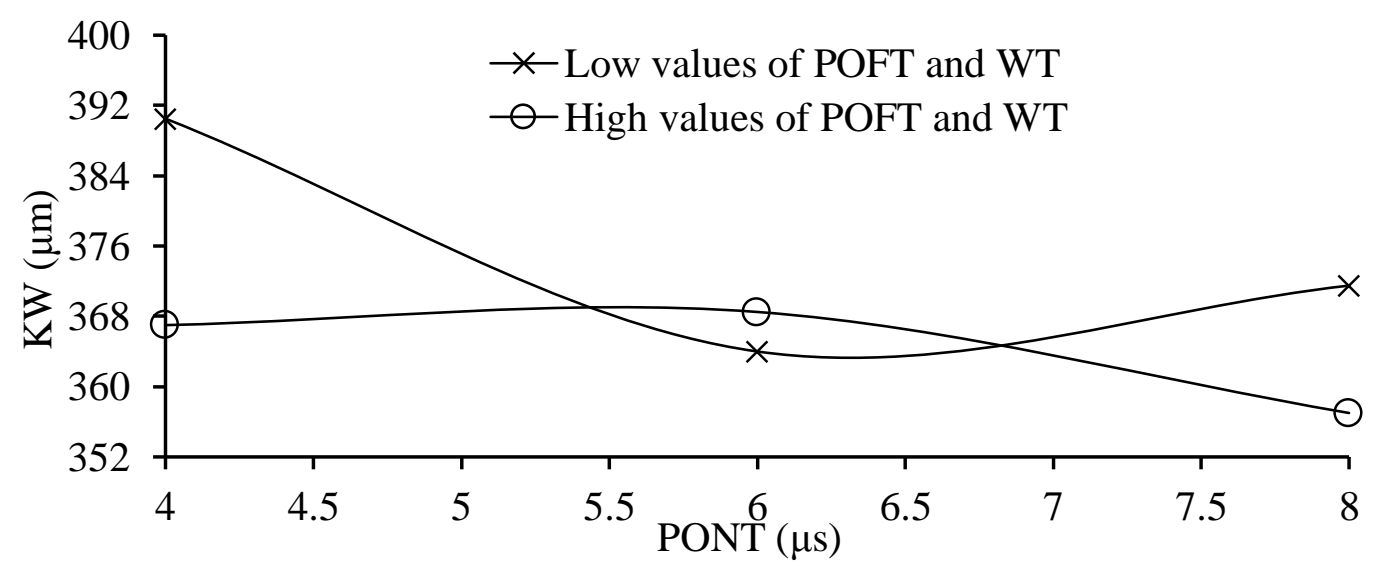

Fig. 2: Effect of PONT on the KW at high and low values of constant parameters.

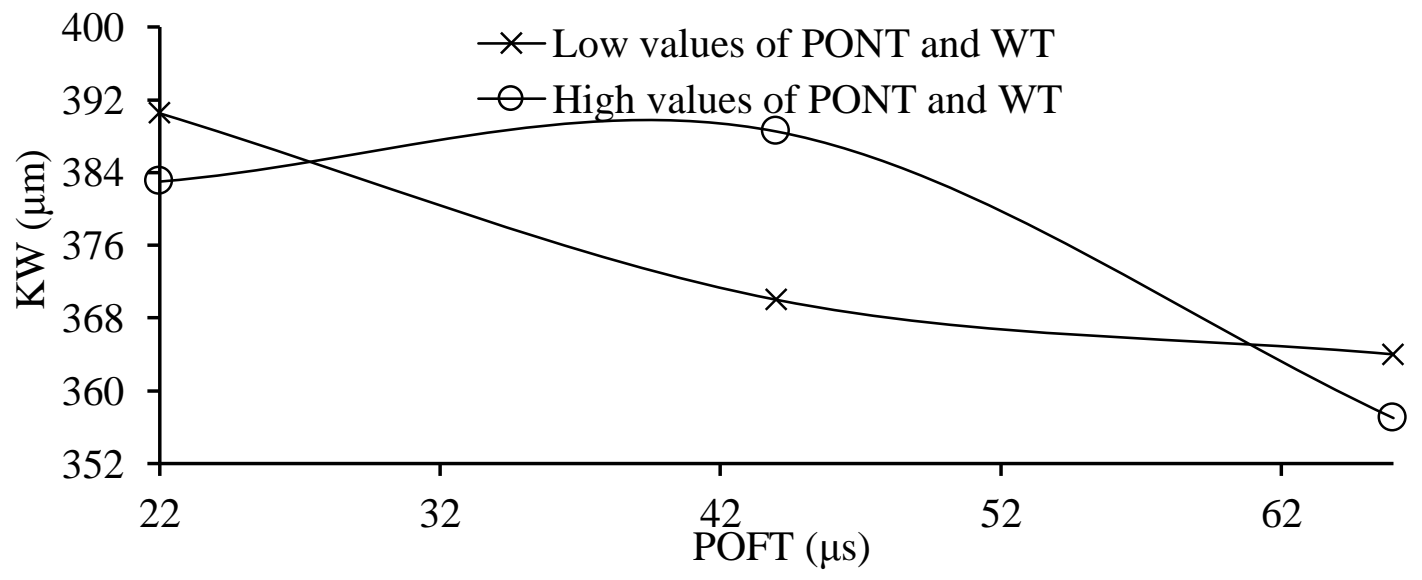

Fig. 3: Effect of POFT on the KW at high and low values of constant parameters. 


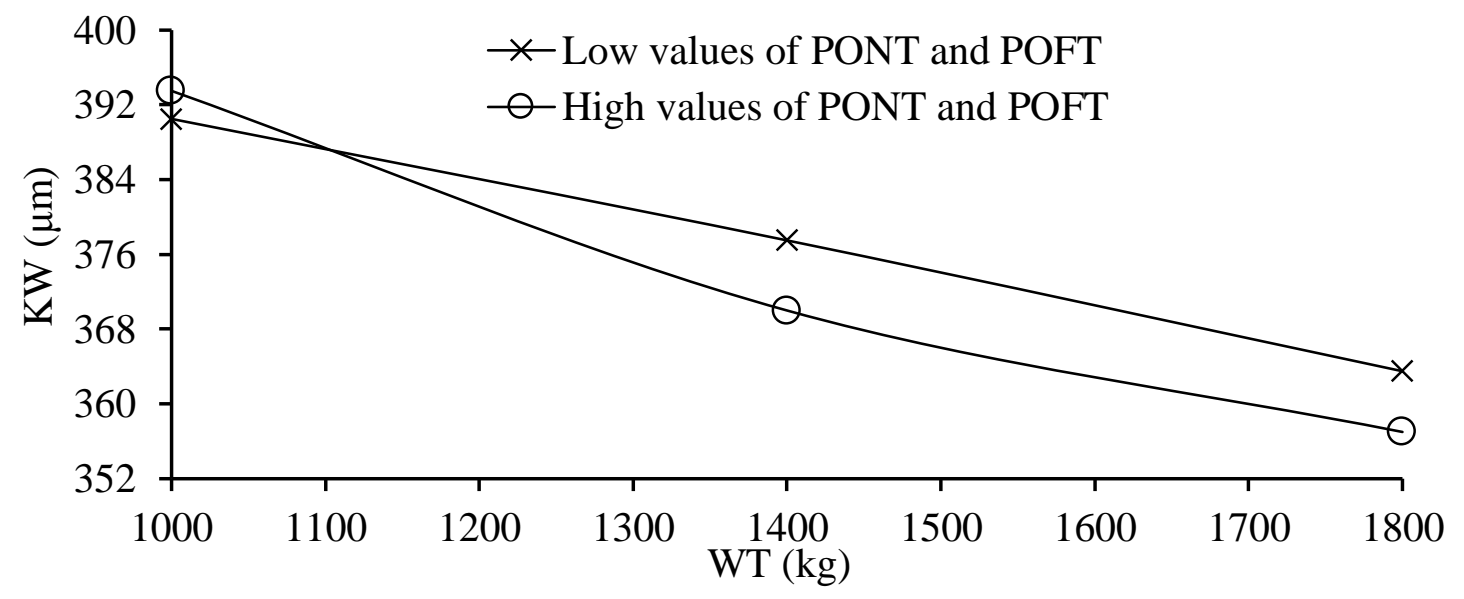

Fig. 4: Influence WT on the KW when constant parameters are of high and low values.

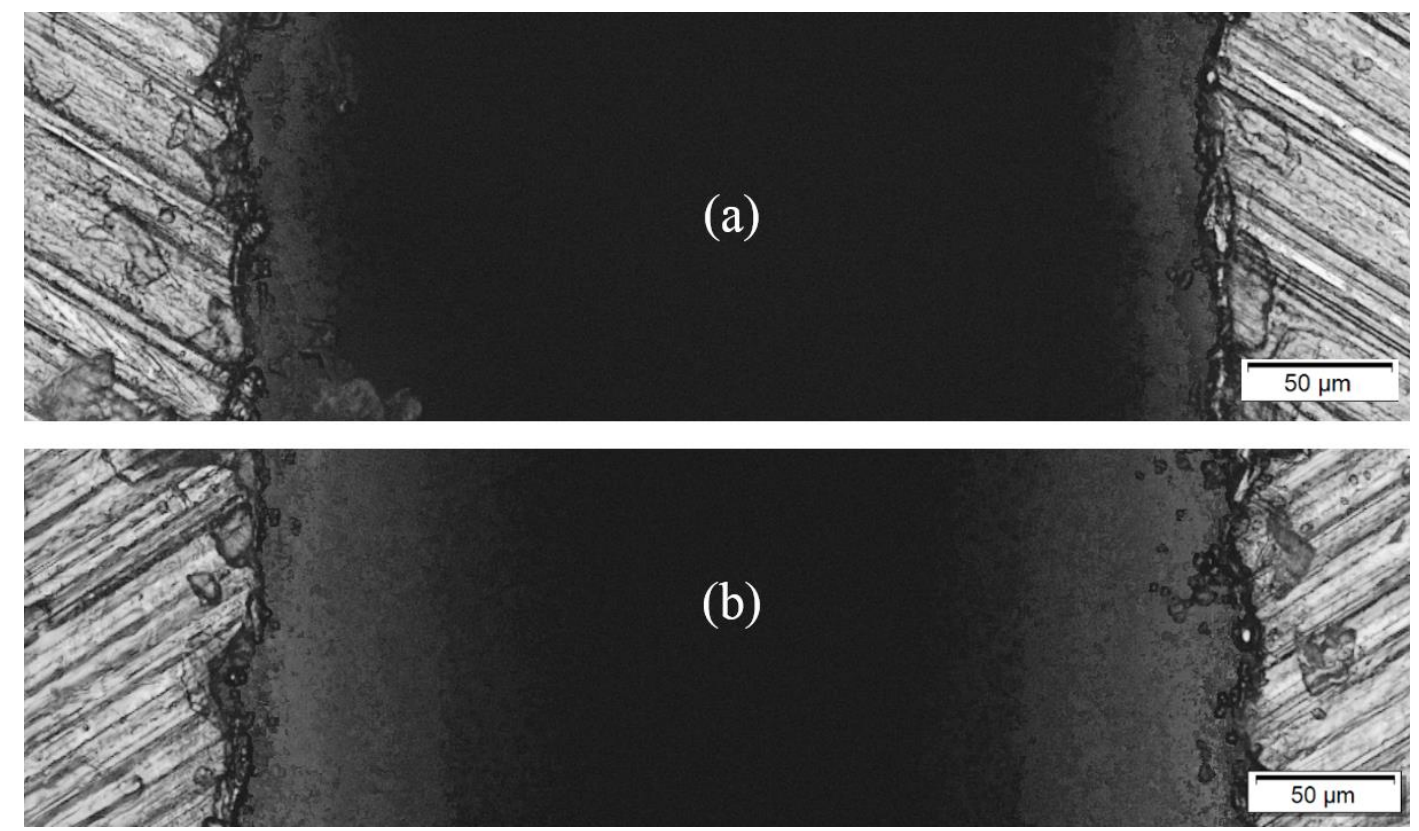

Fig. 5: Kerf generated during WEDM of DSS at $6 \mu$ sONT, $66 \mu$ s POFT and $1800 \mathrm{~kg}$ WT at (a) top and (b) bottom of the workpiece.

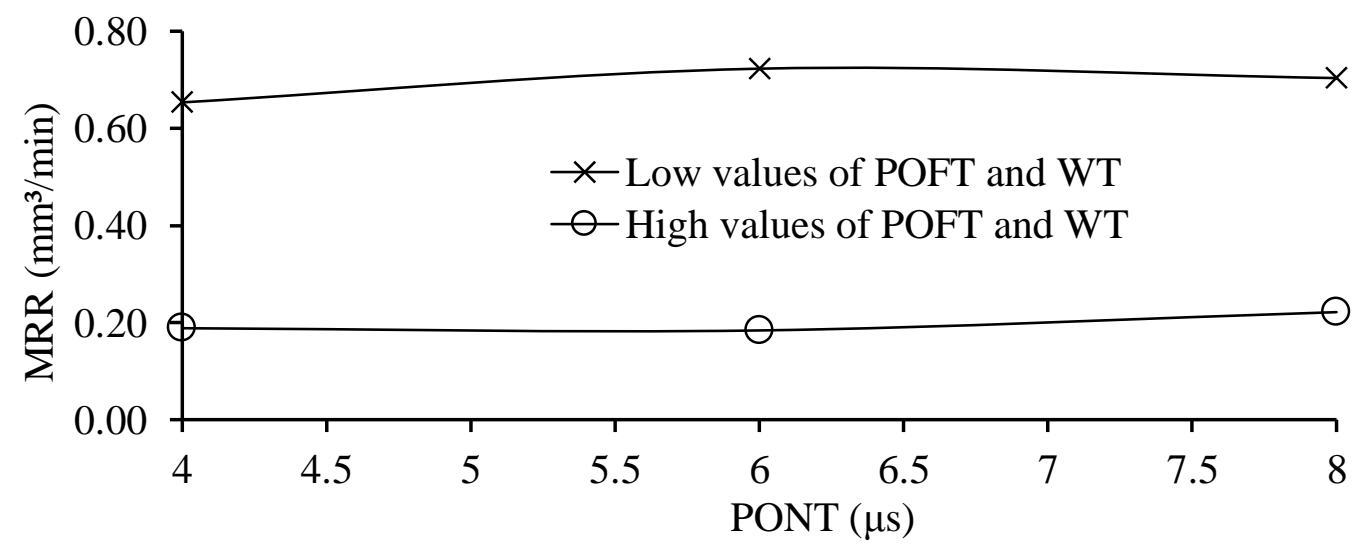

Fig. 6: Effect of PONT on the MRR at high and low values of constant parameters. 


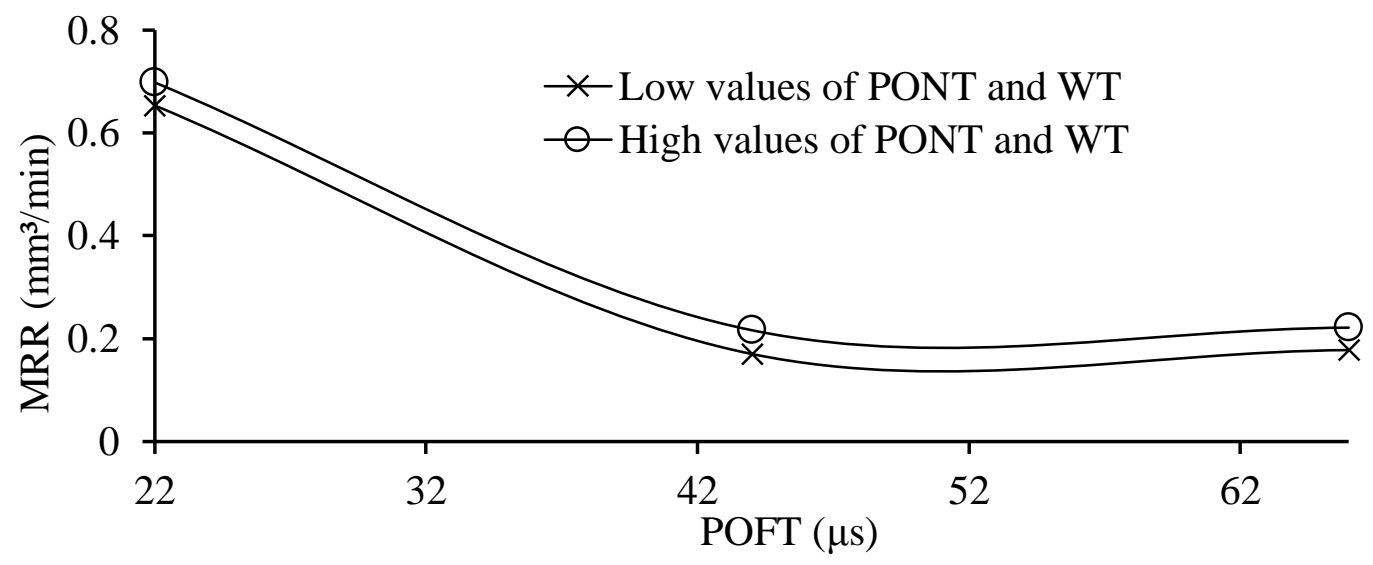

Fig. 7: Effect of POFT on the MRR at high and low values of constant parameters.

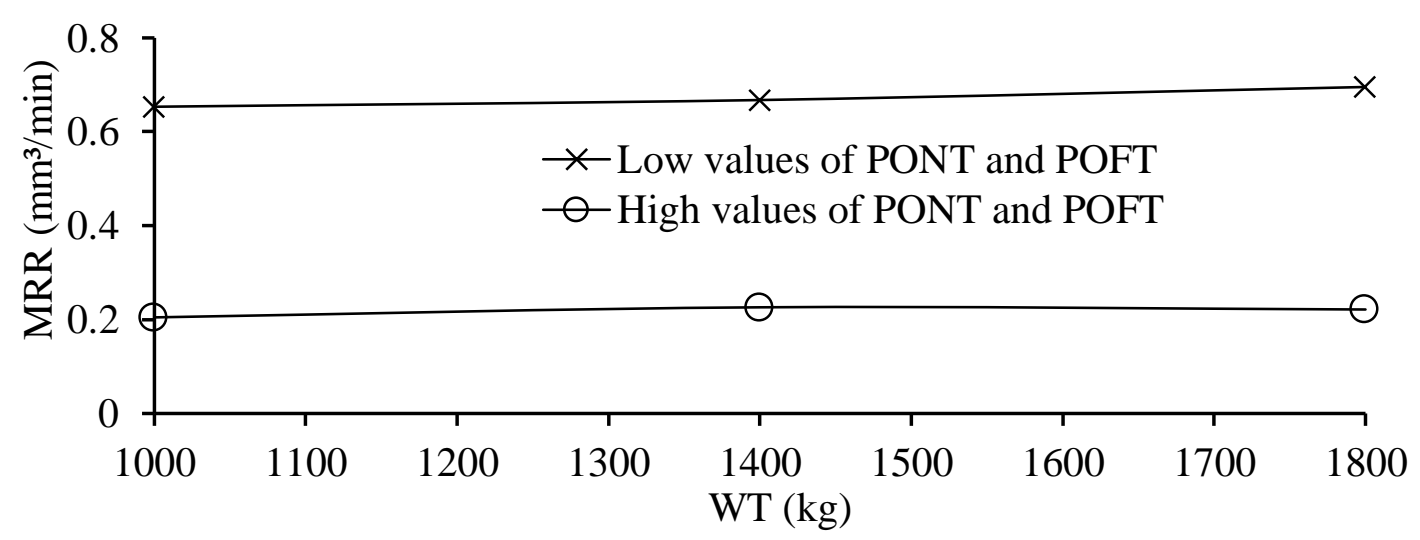

Fig. 8: Influence of WT on MRR at high and low values of constant parameters.

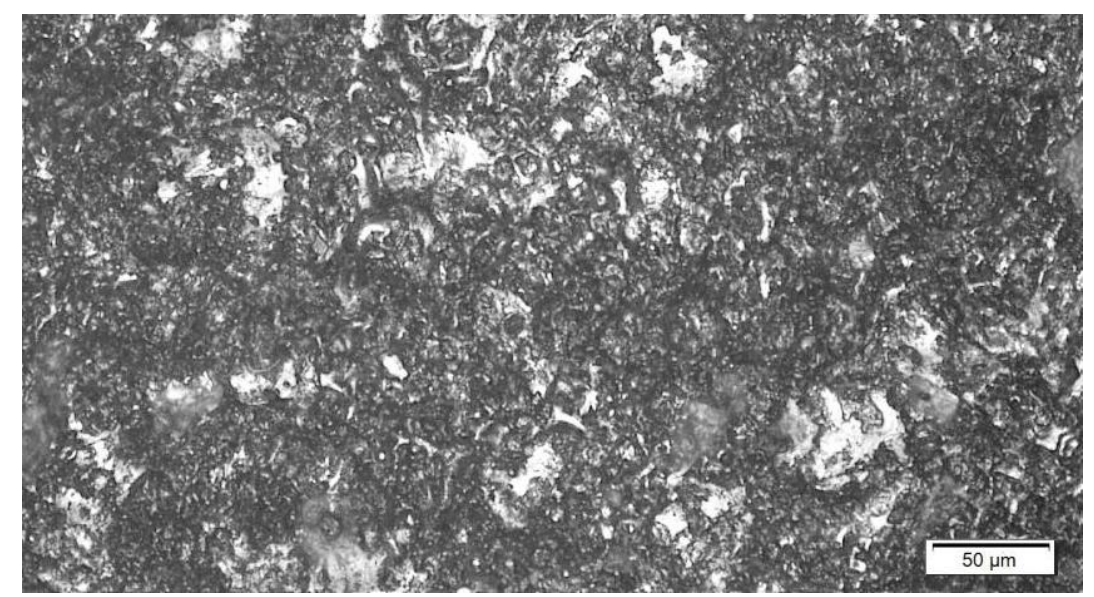

Fig. 9: Surface of 2205 DSS generated from wire EDM. 


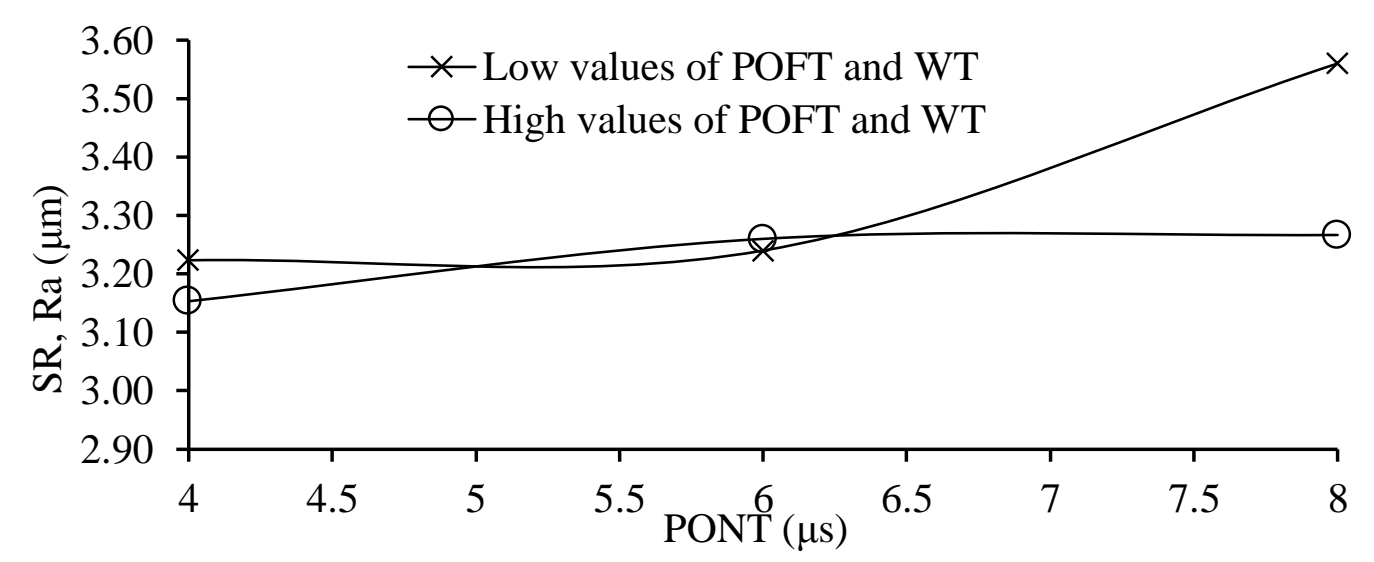

Fig. 10: Influence of PONT on SR at high and low values of constant parameters.

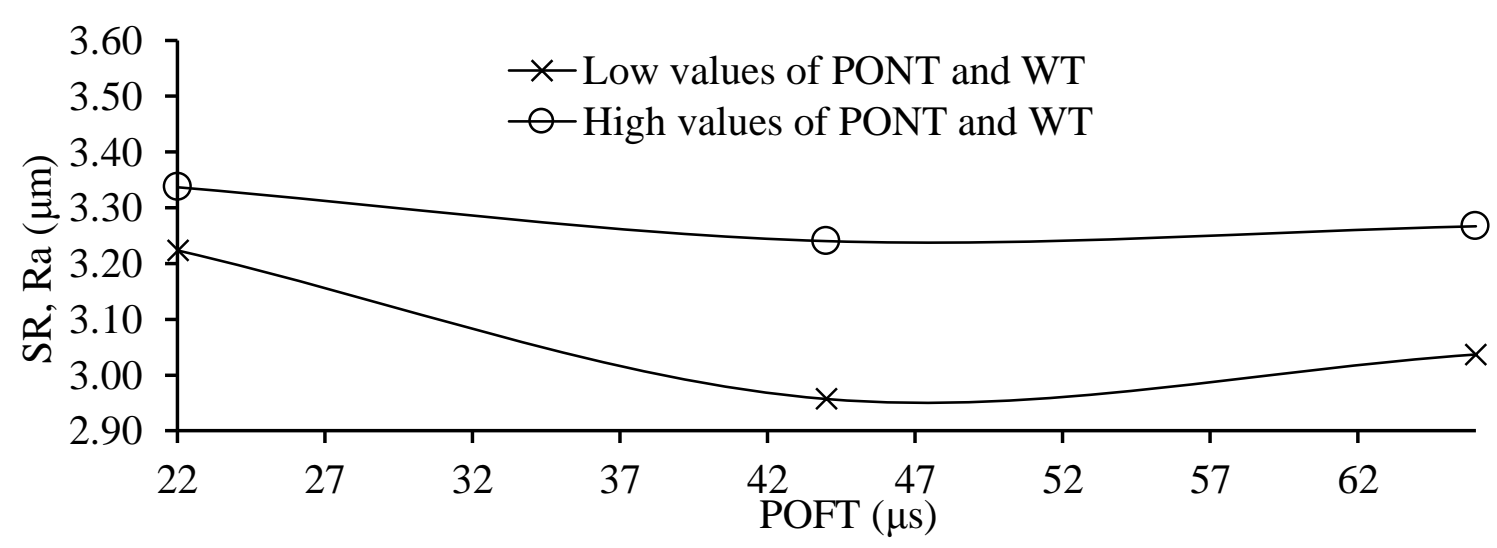

Fig. 11: Influence of POFT on SR at high and low values of constant parameters.

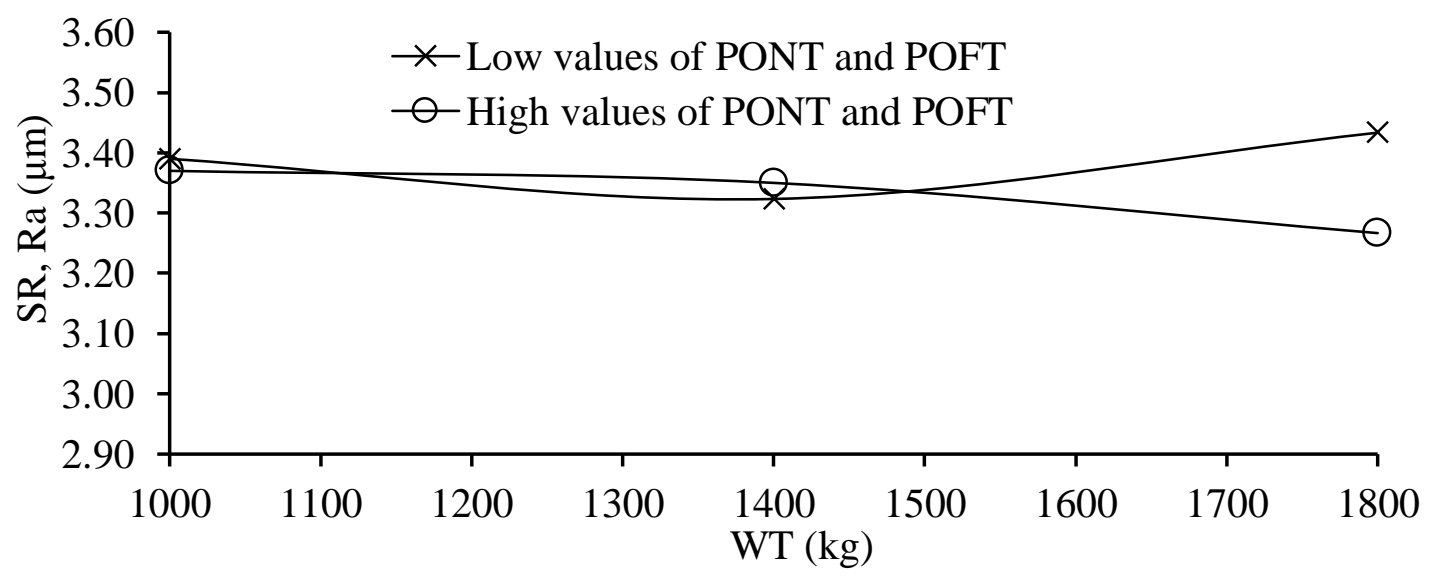

Fig. 12: Influence of WT on SR at different values of constant parameters. 


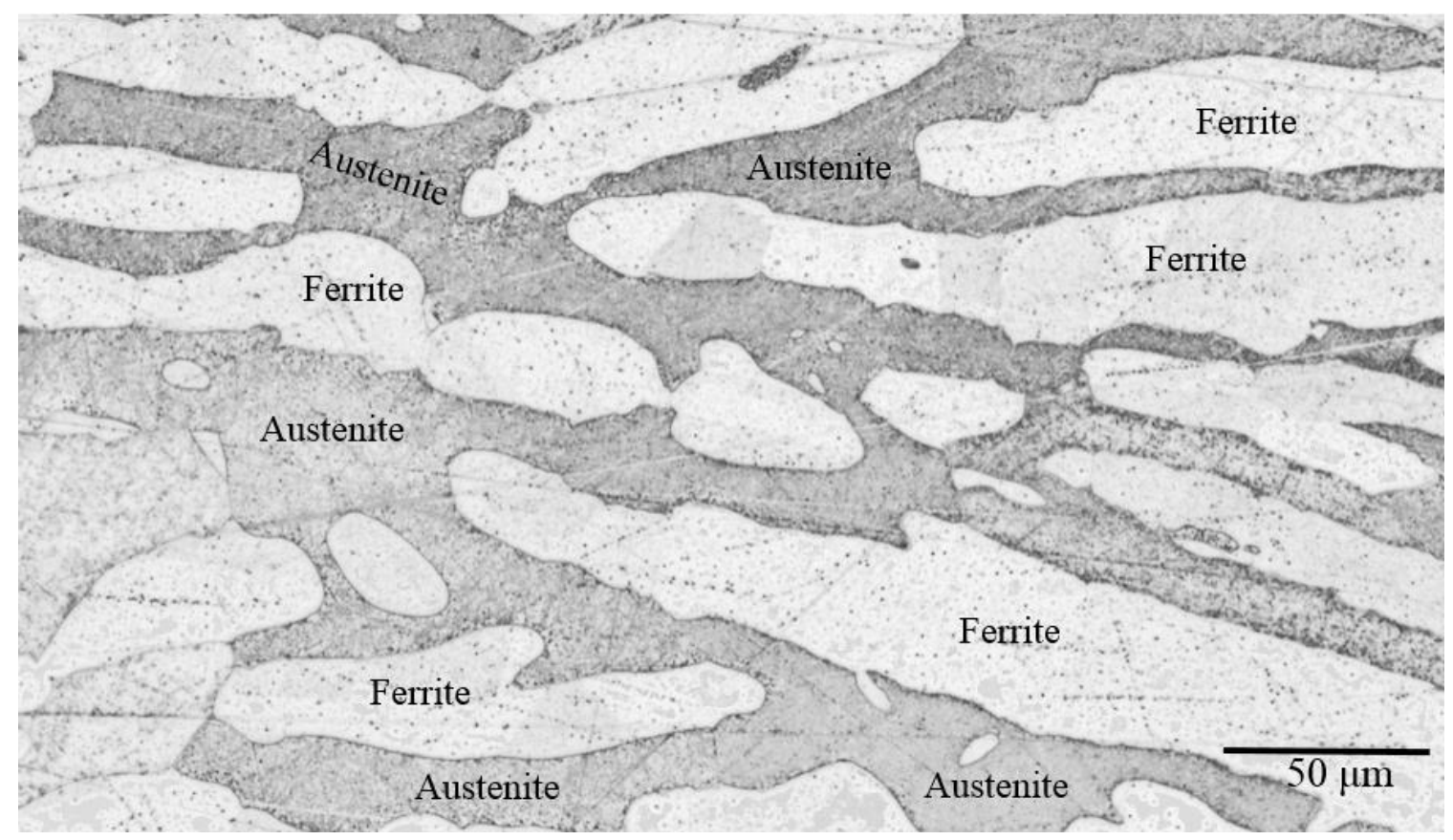

Fig. 13: Structure of DSS before machining.
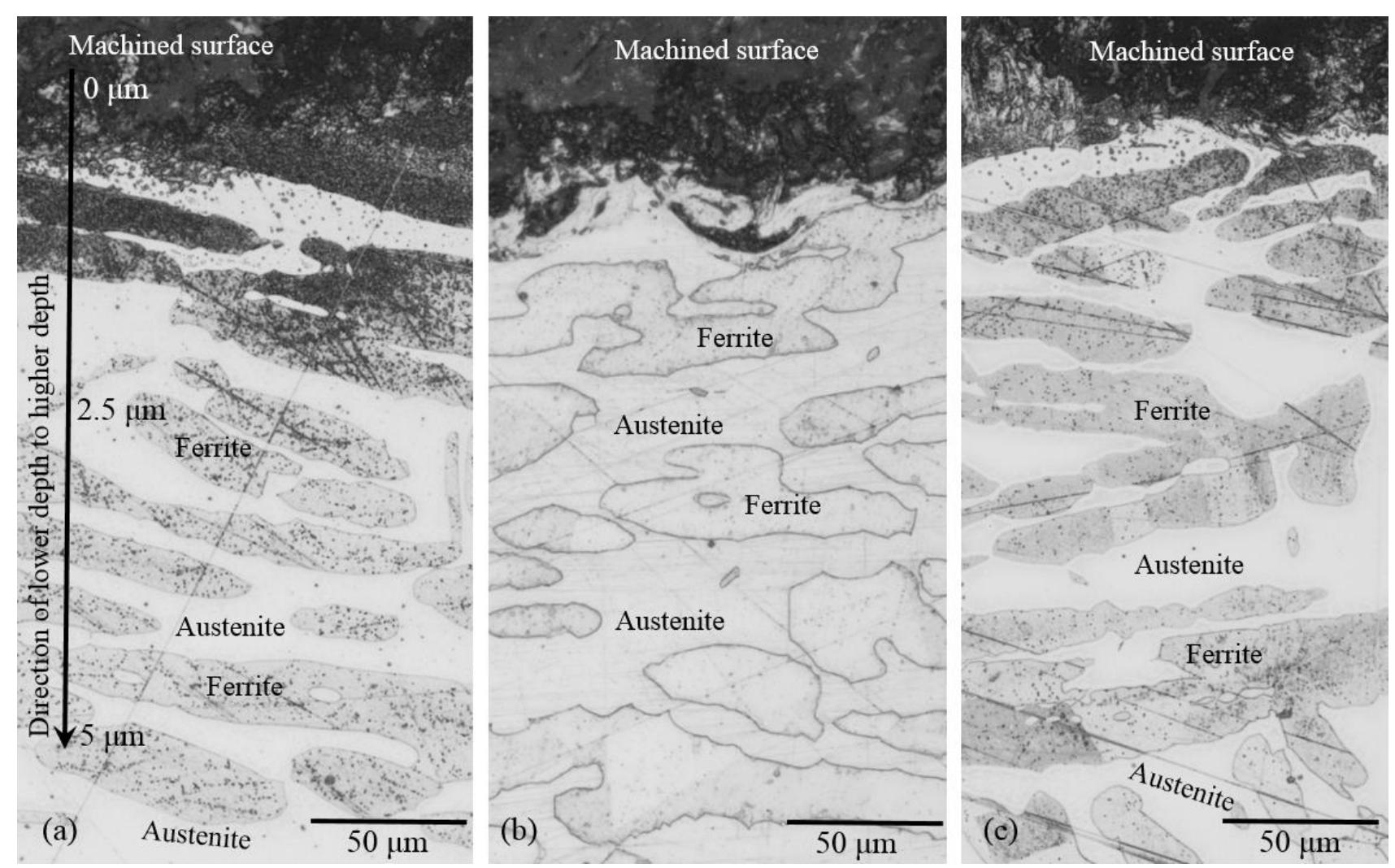

Fig. 14: Microstructure of machined DSS at diverse conditions: (a) PONT $4 \mu \mathrm{s}$, POFT $44 \mu \mathrm{s}$, WT $1000 \mathrm{~kg}$, (b) PONT $8 \mu \mathrm{s}$, POFT $22 \mu \mathrm{s}$, WT $1400 \mathrm{~kg}$ and (c) PONT $8 \mu \mathrm{s}$, POFT $66 \mu \mathrm{s}$ and WT $1400 \mathrm{~kg}$. 


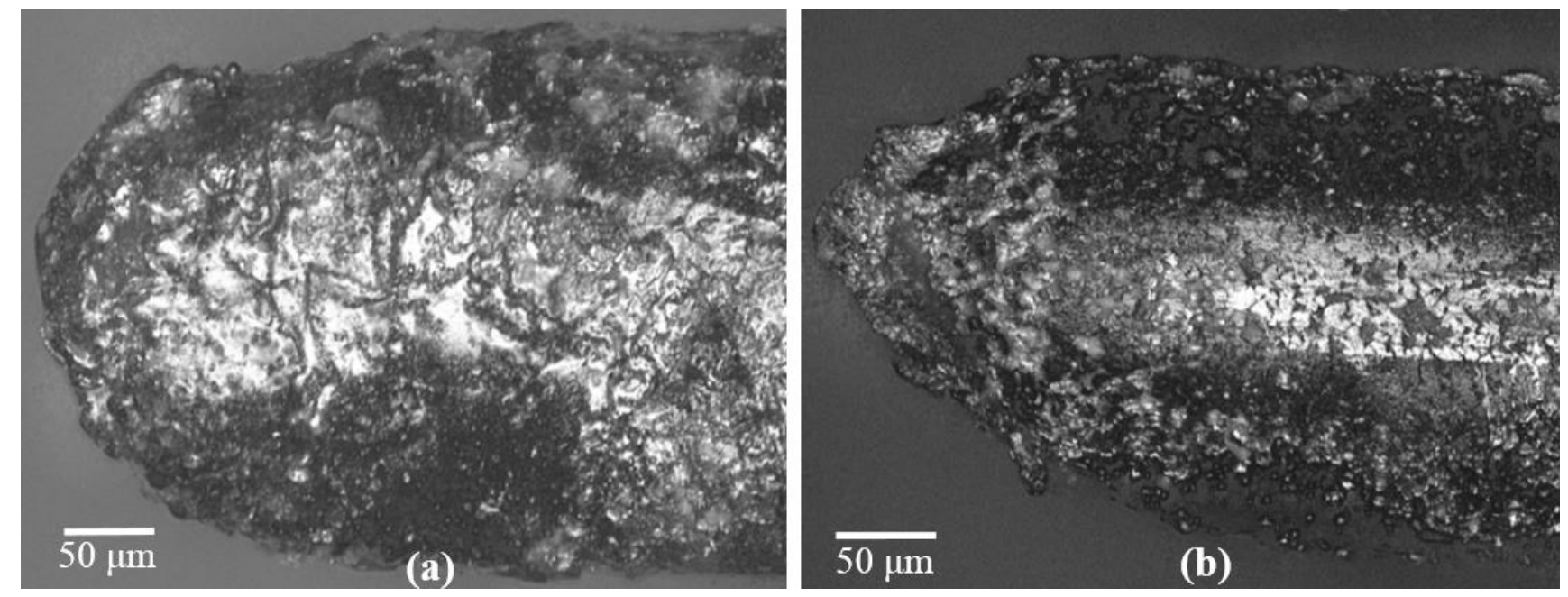

Fig. 15: Broken tips of the wire electrode during WEDM of 2205 DSS. 